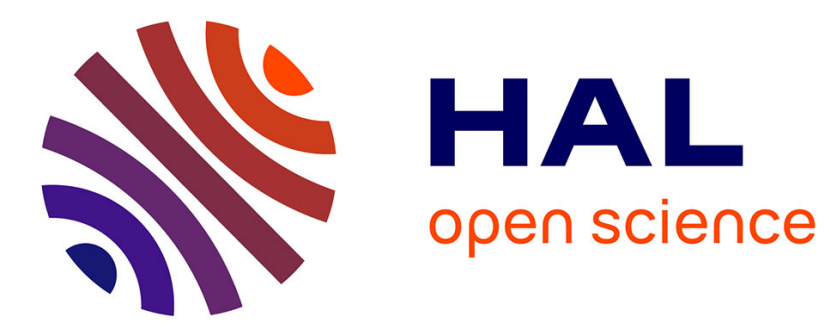

\title{
Facteurs agro-climatiques influençant la résistance stomatique de l'aubergine (Solanum melongena L.)
}

Marie-Christine Daunay, Philippe Malet, Paul-Gérard Schoch, J.-C. L'Hotel, J.-P. Rieu, Evelyne Jullian

\section{- To cite this version:}

Marie-Christine Daunay, Philippe Malet, Paul-Gérard Schoch, J.-C. L'Hotel, J.-P. Rieu, et al.. Facteurs agro-climatiques influençant la résistance stomatique de l'aubergine (Solanum melongena L.). Agronomie, 1986, 6 (7), pp.615-622. hal-00884916

\section{HAL Id: hal-00884916 https://hal.science/hal-00884916}

Submitted on 1 Jan 1986

HAL is a multi-disciplinary open access archive for the deposit and dissemination of scientific research documents, whether they are published or not. The documents may come from teaching and research institutions in France or abroad, or from public or private research centers.
L'archive ouverte pluridisciplinaire HAL, est destinée au dépôt et à la diffusion de documents scientifiques de niveau recherche, publiés ou non, émanant des établissements d'enseignement et de recherche français ou étrangers, des laboratoires publics ou privés. 


\title{
Facteurs agro-climatiques influençant la résistance stomatique de l'aubergine (Solanum melongena L.)
}

\author{
Marie-Christine DAUNAY, Philippe MALET $\left({ }^{*}\right) \&$ Paul-Gérard SCHOCH $(*)$ \\ avec la collaboration technique de J.-C. L'Hotel $\left({ }^{*}\right)$, J.-P. RIEU $\left({ }^{*}\right)$ \& Evelyne JuLLIAN
}

I.N.R.A., Station d'Amélioration des Plantes maraîchères*, Station de Bioclimatologie, Centre de Recherches d'Avignon, F 84140 Montfavet

\begin{abstract}
Chez l'aubergine, du moins chez 3 variétés extrême-orientales et 3 méditerranéennes, la résistance stomatique de la face inférieure des feuilles reste toujours faible même sous un éclairement de 50 à $100 \mathrm{~W} . \mathrm{m}^{-2}$; l'essentiel de la transpiration se fait probablement par la face inférieure des feuilles.

Une expérience de greffage inter-écotypique montre que les racines ont une légère influence sur la résistance stomatique des feuilles quand le sol est maintenu très humide.

En chambre climatisée, les résistances stomatiques des variétés d'aubergine extrême-orientales sont significativement inférieures à celles des variétés méditerranéennes lorsque les températures de l'air sont inférieures ou égales à $25^{\circ} \mathrm{C}$, c'est-à-dire dans les conditions thermiques qui prévalent pour des cultures sous serres. Par contre, aux températures supérieures - plus proches de celles qu'on trouve en culture estivale de plein champ la différence de résistance stomatique entre les 2 écotypes s'atténue pour devenir nulle à $35^{\circ} \mathrm{C}$. Des mesures sur des cultures de plein champ ont confirmé ce résultat.

Finalement, en culture irriguée de plein champ, les variétés méditerranéennes transpirent plus que les extrêmeorientales à cause de leur surface foliaire totale plus grande. Le surplus d'eau nécessaire est fourni grâce à leur système racinaire beaucoup plus développé. Cette meilleure exploitation des ressources en eau est la clef de leur rendement supérieur en culture irriguée de plein champ sous climat méditerranéen.
\end{abstract}

Mots clés additionnels : Eclairement, température, humidité, racines.

\begin{abstract}
Leaves of three eggplant varieties from the Far East and three from the Mediterranean always had low abaxial stomatal resistance, even under $50-100 \mathrm{~W} \cdot \mathrm{m}^{-2}$ light (fig. 1). Therefore, the major part of plant transpiration passed through the abaxial surface of leaves. An inter-ecotype grafting experiment showed that roots have a slight influence on leaf stomatal resistance if the soil is kept wet (fig. 4). In a climate chamber, eggplant varieties from the Far East (EO in text) had significantly lower stomatal resistances than those of Mediterranean varieties (M) at air temperatures less than $25^{\circ} \mathrm{C}$, i.e. under temperature conditions common in greenhouses. At higher temperatures closer to those prevailing in the field under summer irrigation, these differences between the two ecotypes decreased until by $35^{\circ} \mathrm{C}$ they had completely disappeared (fig. 3 ). This result has been confirmed by measurements in the field. Finally irrigated Mediterranean varieties in the open transpire more than their Far eastern counterparts by virtue of their greater leaf area per plant. This is possible because of their larger root systems which give access to a greater volume of stored soil moisture. This better exploitation of water resources is the key to their higher yield capacity under irrigation in a Mediterranean climate.
\end{abstract}

Additional key words : Light, temperature, humidity, roots.

\section{INTRODUCTION}

Nombre de caractéristiques morphologiques et physiologiques des variétés d'aubergine sont déterminées par leur aire agro-climatique d'origine. Ainsi par rapport aux variétés méditerranéennes $(\mathrm{M})$, celles
d'Extrême-Orient (EO) sont généralement plus anthocyanées et plus précoces; leurs racines sont moins denses et moins profondes (DAUNAY, 1982) et leurs fréquences stomatiques - indice et densité plus faibles sur les faces supérieures des feuilles (DAUNAY et al., 1986). 
En enceintes climatisées et sous serres (POCHARD \& SERIEYS, 1974 ; POCHARD, 1979), les jeunes plants d'origine extrême-orientale transpirent plus que les méditerranéens et ce résultat correspond à des résistances stomatiques plus faibles chez les variétés EO. Par ailleurs, une expérience complémentaire de greffage intervariétal laisse à penser que les niveaux de transpiration dépendent exclusivement des greffons et de leur feuillage et non des porte-greffes et de leurs racines.

Mais au champ, avec des doses d'irrigation convenables (COLLECTIF, 1975), la résistance stomatique de la face inférieure des feuilles est extrêmement faible pendant toute la phase diurne, quelle que soit la variété, si bien que la différence entre les 2 écotypes disparaît complètement. A la face supérieure, il existe une régulation stomatique un peu plus active; mais il est probable que la résistance stomatique « moyenne » des feuilles est essentiellement déterminée par celle de la face inférieure : hypothèse confirmée par des travaux récents (SCHOCH, non publiés).

Pour comprendre pourquoi les 2 écotypes ont (au champ) ou n'ont pas (en serre et en enceinte climatisée) des résistances stomatiques identiques, nous analyserons séparément les effets de l'éclairement, de la température et de l'humidité de l'air. De plus, nous utiliserons des plantes intergreffées pour vérifier que les porte-greffes n'ont pas d'influence sur les résistances stomatiques. L'échantillonnage variétal sera composé de 3 variétés représentatives de chacun des écotypes, méditerranéen et extrême-oriental.

\section{MATÉRIELS ET MÉTHODES}

\section{A. Matériel végétal}

\section{Au champ}

\section{2 types d'essais sont réalisés :}

Avec des plantes entières (1981), 3 variétés de chaque écotype sont comparées : pour l'extrême-oriental, "Liu Yé Qié » (LIU), «Ronde de Valence » (RVA) et "Shinkuro» (SHI); pour le méditerranéen «LF3-24» (LF3), «Dourga » (DOU) et « Jordanie» (JOR). La plantation (après semis, repiquage en pots et élevage des plants sous tunnel plastique) est effectuée fin mai, au stade 7 à 9 feuilles.

Pour les plantes greffées (1982), le greffage a lieu en serre au stade 6 à 7 feuilles, selon la technique décrite par BEYRIES (1979). La plantation intervient 2 à 3 semaines plus tard. Six des 18 combinaisons « inter-écotypique » possibles sont comparées simultanément (tabl. 1).

\section{En enceinte climatisée (1980-1982)}

\section{Plusieurs essais sont réalisés :}

Avec les plantes entières, selon la place disponible au moment de chaque essai, 1, 2 ou 3 couples variétaux inter-écotypiques sont comparés. Le transfert des plantes depuis les serres (où elles sont préalablement élevées), se fait au stade 5 à 6 feuilles étalées, au moins $24 \mathrm{~h}$ avant le début des mesures.
TABLEAU 1.

Formules de greffage.

Grafting treatments.

\begin{tabular}{|c|c|c|c|c|}
\hline \multicolumn{2}{|c|}{ Ecotype } & \multicolumn{2}{|c|}{ Enceinte climatisée } & \multirow{2}{*}{$\begin{array}{l}\text { Champ } \\
\text { formule } \\
\text { de greffe }\end{array}$} \\
\hline greffon & $\begin{array}{l}\text { porte- } \\
\text { greffe }\end{array}$ & $\begin{array}{c}\text { essai } \\
n^{\circ}\end{array}$ & $\begin{array}{c}\text { formule } \\
\text { de greffe }\end{array}$ & \\
\hline \multirow[t]{10}{*}{$\mathrm{EO}$} & EO & 1 & RVA/RVA (a) & RVA/RVA (a) \\
\hline & M & & RVA/DOU & RVA/DOU \\
\hline & EO & 2 & RVA/RVA (a) & - \\
\hline & $\mathrm{M}$ & & RVA/LF3 & - \\
\hline & EO & 3 & LIU/LIU (a) & LIU/LIU (a) \\
\hline & M & & LIU/LF3 & $\mathrm{LIU} / \mathrm{LF} 3$ \\
\hline & $\mathrm{EO}$ & 4 & LIU/LIU (a) & -- \\
\hline & M & & LIU/DOU & - \\
\hline & $\mathrm{EO}$ & - & - & SHI/SHI (a) \\
\hline & $\mathrm{M}$ & & - & $\mathrm{SHI} / \mathrm{JOR}$ \\
\hline \multirow[t]{10}{*}{ M } & $\mathrm{M}$ & 1 & DOU/DOU (a) & $\mathrm{DOU} / \mathrm{DOU}(\mathrm{a})$ \\
\hline & EO & & DOU/RVA & DOU/RVA \\
\hline & $\mathbf{M}$ & 2 & LF3/LF3 (a) & - \\
\hline & EO & & LF3/RVA & - \\
\hline & $\mathrm{M}$ & 3 & LF3/LF3 (a) & LF3/LF3 (a) \\
\hline & EO & & LF3/LIU & $\mathrm{LF} 3 / \mathrm{LIU}$ \\
\hline & M & 4 & DOU/DOU (a) & - \\
\hline & EO & & $\mathrm{DOU} / \mathrm{LIU}$ & - \\
\hline & M & - & - & JOR/JOR (a) \\
\hline & EO & & - & JOR/SHI \\
\hline
\end{tabular}

a : Témoins autogreffés; control.

Pour les plantes greffées, 8 combinaisons de greffage sont mises en comparaison (tabl. 1), au moyen de 4 essais successifs. Le transfert en enceinte climatisée intervient au moment de la floraison du $3^{e}$ étage floral du greffon. Les fruits sont supprimés au fur et à mesure de leur formation, pour limiter la consommation en eau des plantes (car la réserve disponible dans un pot est limitée) et pour éviter l'opération de tuteurage.

\section{Sous serre (1981)}

L'expérience d'ombrage diminuant l'éclairement (cf. § II.B) est mise en place au-dessus de « Dourga » (M) et de "Ronde de Valence » (EO), cultivées en pots et bien arrosées.

\section{B. Conditions agro-climatiques et dispositifs expé- rimentaux}

Les essais au champ sont irrigués 2 fois par semaine (alternativement par gravité et par aspersion), les doses d'irrigation compensant l'évapotranspiration potentielle calculée, suivant la formule de PENMAN, à partir des relevés de la station météorologique de l'INRA d'Avignon. Il s'agit de dispositifs en blocs : 5 blocs contenant 9 plantes de chaque variété, pour l'essai avec des plantes entières, 3 blocs contenant 3 plantes de chaque formule de greffage dans l'autre essai. Au cours des 2 étés d'expérience, 8 journées de mesure sont choisies avec un bon ensoleillement, très peu de vent, pour moitié juste avant et, pour moitié, juste après une irrigation. Les températures maximales de ces journées sont comprises entre $28^{\circ}$ et $35^{\circ} \mathrm{C}$. 
Les chambres climatisées sont utilisées pour faire varier la température et l'humidité de l'air. L'éclairement $\left(80 \mathrm{~W} . \mathrm{m}^{-2}\right)$ et la photopériode $(12 \mathrm{~h} / 12 \mathrm{~h})$ sont toujours restés constants. Les plantes de chaque variété (de 6 à 20 plantes suivant les essais) y sont réparties au hasard. Les résultats présentés plus loin sont les moyennes des mesures effectuées le $3^{\mathrm{e}}$ jour après le transfert des plantes dans la chambre ; car des expériences préliminaires ont montré que ce délai d'adaptation est nécessaire pour que les différences variétales s'établissent définitivement (DAUNAY, non publié).

Pour étudier l'influence du niveau d'éclairement, 2 densités d'ombrage sont créées dans une serre, au moyen de filets plastiques; d'après des mesures comparatives du rayonnement global et des enregistrements de spectres lumineux, ces ombrages ont assuré, dès le repiquage des plantes, 75 et 50 p. 100 de l'éclairement normal de la serre, sous lequel se trouve aussi un lot témoin des 2 variétés en comparaison. Avec un coefficient de transmission de la lumière d'environ 80 p. 100, d'après les mesures de MERMIER (comm. pers., 1984) sous des serres semblables, 3 niveaux moyens d'éclairement sont ainsi obtenus : environ 80 , 60 et 40 p. 100 du rayonnement global extérieur.

Les mesures sont faites au cours d'une journée nuageuse (le 11 mars 1981), sous deux niveaux de rayonnement global moyen " extérieur »: $126 \mathrm{~W} \cdot \mathrm{m}^{-2}$ (de 10 à $11 \mathrm{~h} \mathrm{TU}$ ), puis $287 \mathrm{~W} \cdot \mathrm{m}^{-2}$ (de 15 à $16 \mathrm{~h}$ ), avec des températures de l'air interne respectivement de 18,5 et $22^{\circ} \mathrm{C}$. Les mesures sont répétées le lendemain pendant une période ensoleillée (de 15 à $16 \mathrm{TU}$ ) avec en moyenne $356 \mathrm{~W} . \mathrm{m}^{-2}$ à l'extérieur et $24,5^{\circ} \mathrm{C}$ de température de l'air interne. On obtient ainsi 9 niveaux différents de rayonnement global (3 traitements d'ombrage $\times 3$ périodes de mesure), dont les valeurs sont évaluées à partir des coefficients d'abattement de 80,60 , et 40 p. 100 .

\section{Mesures des résistances stomatiques}

L'appareil utilisé est un poromètre Delta Devices. Les mesures sont faites exclusivement sur la face inférieure des feuilles, pour la raison exposée dans l'introduction.

\section{Au champ}

Les feuilles sont systématiquement choisies juste en dessous d'une fleur récemment épanouie, sur 10 plantes entières par variété (ou les 9 plantes possibles pour chaque formule de greffe) et mesurées de $8 \mathrm{~h}$ TU après la disparition de la rosée - au coucher du soleil. En cas de coup de vent au moment d'une mesure, celle-ci est interrompue et éliminée, de façon à conserver le plus possible des conditions comparables d'un traitement à un autre.

\section{En enceinte climatisée}

Les mesures sont faites (i) sur la $3^{\mathrm{e}}$ et/ou la $4^{\mathrm{e}}$ feuille de la tige principale, quand l'essai compare des jeunes plantes de 5-6 feuilles, (ii) sur les feuilles insérées juste en dessous d'une fleur épanouie, quand l'essai compare des plantes plus âgées. Les mesures sont répétées plusieurs fois par jour, toujours sur des feuilles entièrement éclairées.

\section{Sous la serre (avec les 3 traitements d'ombrage)}

Les mesures sont faites sur les feuilles d'ordre 3 , sur 18 plantes au stade 5-6 feuilles pour chacune des 2 variétés en comparaison.

\section{RÉSULTATS}

\section{A. Influence du rayonnement global}

Comme on pouvait s'y attendre, les résistances stomatiques des 2 variétés en comparaison ont tendance à augmenter quand le rayonnement diminue (fig. 1) et, dans la gamme des éclairements inférieurs à $100 \mathrm{~W} . \mathrm{m}^{-2}$, la différence entre les résistances de «Dourga » $(\mathrm{M})$ et de « RVA » (EO) devient systématiquement significative. Cependant le maximum de résistance enregistré pour "Dourga 》 $\left(4,5 \mathrm{~s} \cdot \mathrm{cm}^{-1}\right.$ à $50 \mathrm{~W} \cdot \mathrm{m}^{-2}$ ) correspond encore à une faible régulation stomatique.

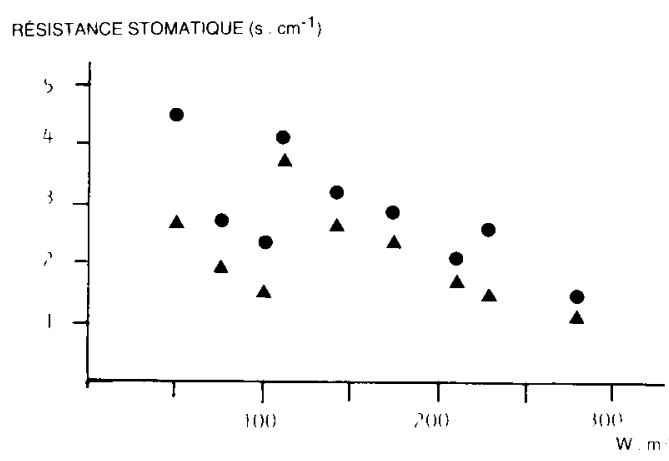

Figure 1

Influence du rayonnement global sous serre.

Influence of global radiation in greenhouse.

- Variété extrême-orientale. Far-East variety.

- Variété méditerranéenne. Mediterranean variety.

\section{B. Influence de la température et de l'humidité de l'air}

\section{Plantes entières}

Les feuilles d'ordre 3 et 4 des plantes jeunes enregistrent toutes les deux les différences variétales (fig. 2), mais la 3 plus sûrement que la 4.

Dans un air presque saturé d'humidité (fig. 3a), la résistance stomatique diminue et les écarts variétaux s'amenuisent quand la température s'élève de 20 à $35{ }^{\circ} \mathrm{C}$; puis, au-delà de $35^{\circ} \mathrm{C}$, elle augmente brutalement, ainsi que les écarts variétaux. En air sec (fig. 3b), elle est plus élevée qu'en air humide, sauf à $22^{\circ} \mathrm{C}$, si bien que les différences variétales sont généralement un peu plus nettes. En dessous de $25^{\circ} \mathrm{C}$, l'amplitude des différences variétales est tamponnée, sans doute parce que la différence d'humidité de l'air (80 contre $95 \mathrm{p}$. 100) représente une variation du déficit de saturation $(3,9-0,9=3 \mathrm{mb})$ nettement plus faible qu'aux températures supérieures (à $31^{\circ} \mathrm{C}$ : 

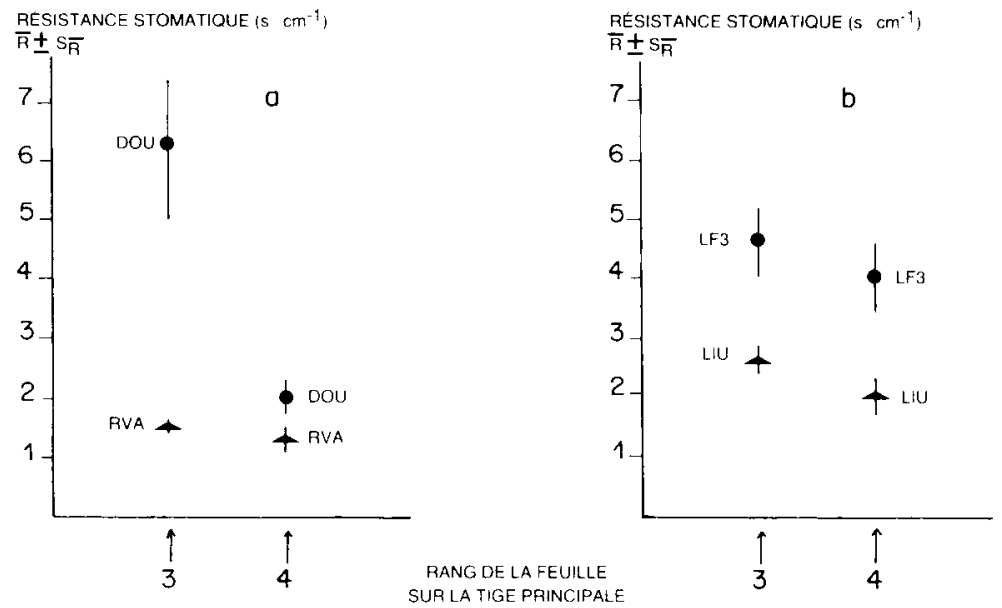

Figure 2

Résistance stomatique en enceinte climatisec' : influence de l'äge dess feuilles ttempérature: $22{ }^{\circ} \mathrm{C}$. humidlité relative de l'air : $80 \mathrm{p}$. 100$)$.

Stomatal resistance in climatic chamber : influence of leaf age 1 tem perature: :2 "C, air relative humidity : $80 \% \%)$. a: Varietes $R V A$ et DOU. Varieries $R V A$ and $D O U$.

b: Varietés $L I U$ et LF3. Varieties LIU and LF3.

A Variété extrême-orientale. Far-East variety.

- Variété méditerranéenne. Mediterranean variety.

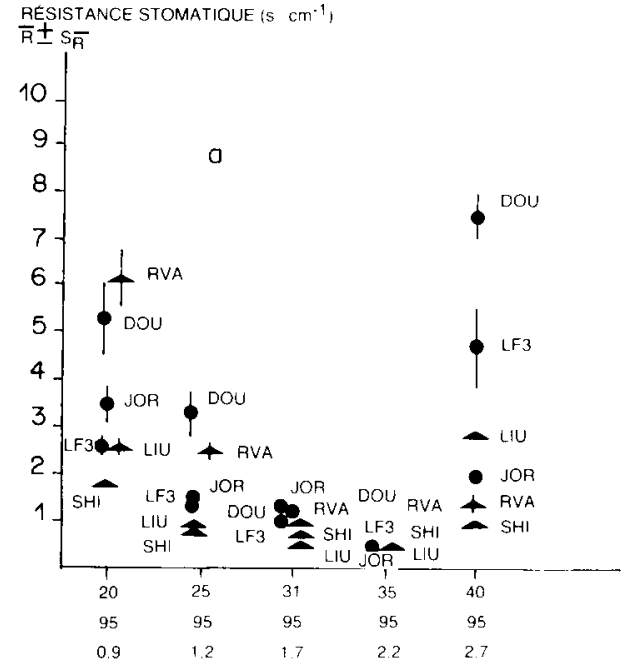

Figure 3

Résistance stomatique sur la 3r feutlle en enceinte climatisee: influence de la temperature $(T)$, de l'humidite relative $(H) .100)$ et diu déficit de' saturation (DS) de l'air.

Stomatal resistance on the third leaf in climatic chamber : influence of air temperature $(T)$, relative humidits $(H \%)$ and saturation defi (it (DS).

$8,3 \mathrm{mb}$ ). Au-delà de $35^{\circ} \mathrm{C}$, la réponse au déficit de saturation dépend des variétés et surtout des écotypes : la résistance des variétés EO est légèrement plus élevée en air sec qu'en air humide, alors que celle des variétés M (LF3 et surtout DOU) est plus faible.

En bilan, les 2 variétés typiquement extrêmeorientales, LIU et SHI, se distinguent des méditerranéennes, en particulier de LF3 et DOU, par des résistances stomatiques généralement plus faibles et par des réactions de plus faible amplitude aux variations de la température et du déficit de saturation. Par contre RVA (EO), qui est implantée depuis longtemps en région méditerranéenne, se rapproche des variétés (M) par une réactivité nettement plus ample aux variations de température et d'humidité de l'air.

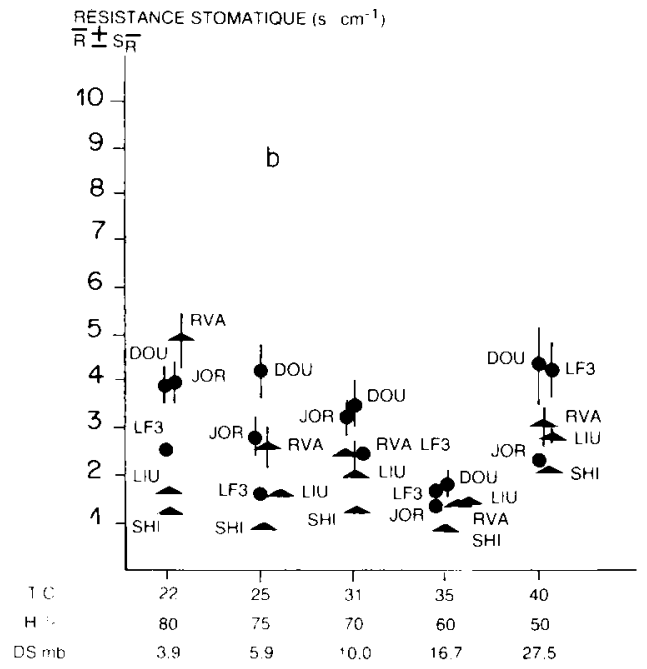

a: Temperature variahle. Variahle tomperature.

$b$ : Température et $H \%$ variables. Variable temperature and $H \%$.

- Variété extrême-orientale. Far-East variety.

- Variété méditerranéenne. Mediterranean variety.

\section{Plantes greffées}

On peut distinguer 2 effets du greffage sur la résistance stomatique: celui du changement des racines sous une partie aérienne constante (fig. 4) et celui du changement des parties aériennes sur un système racinaire constant (fig. 5).

Les racines ont une influence certaine sur la résistance stomatique des feuilles (fig. 4): les portegreffes $\mathrm{M}$ (DOU et LF3) ont tendance à augmenter la résistance stomatique des variétés EO (fig. 4a) et, inversement, les porte-greffes EO (RVA et LIU) à diminuer celle des variétés $M$ (fig. 4b). Cependant, les différences ne sont pas toutes significatives.

L'influence du feuillage est tout de même prédomi- 


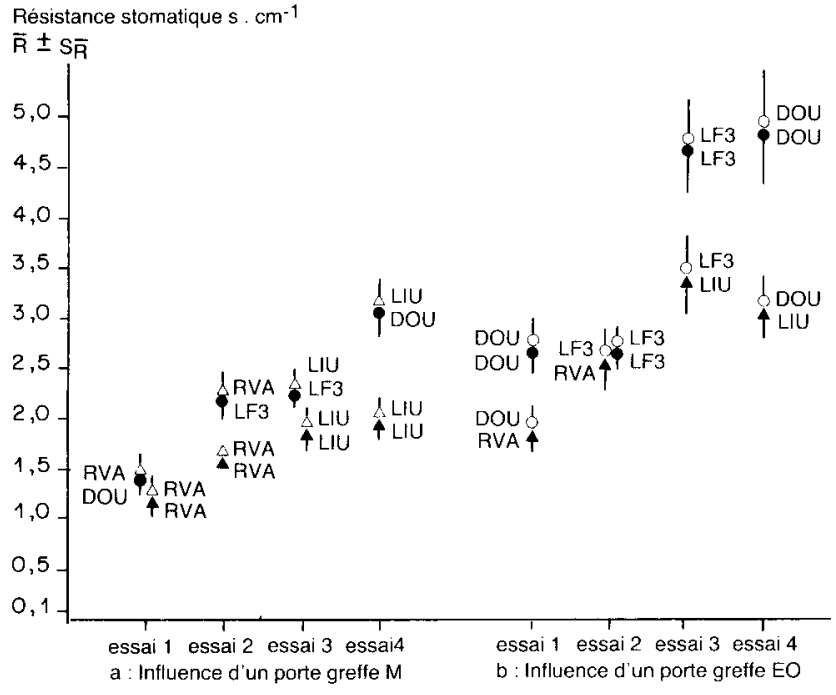

Figure 4

Résistance stomatique en enceinte climatisée : influence des portegreffe (signe du bas)

(temperature : $26{ }^{\circ} \mathrm{C}$, humidite relative : $\left.80 \mathrm{p} .100\right)$.

Stomatal resistance in climatic chamber : influence of root-stocks. (lower sign)

(temperature: $26{ }^{\circ} \mathrm{C}$, relative humidity : $80 \%$ ).

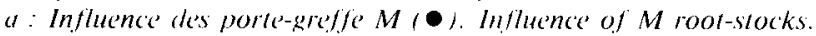

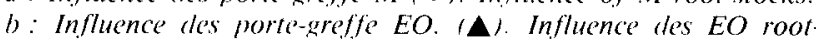
stocks.

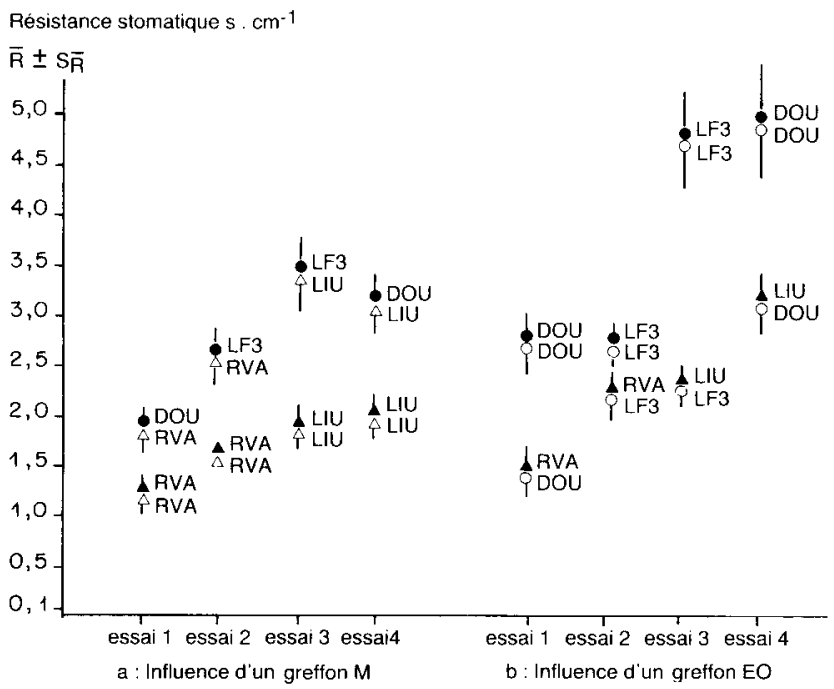

Figure 5

Résistance stomatique en enceinte climatisée : influence des greffons (signe du haut).

Stomatal resistance in a climatic chamber: influence of scions (upper sign).

$a$ : Influence des greffons $M(\bullet)$. Influence of $M$ scions.

$b$ : Influence des greffons $E O(\mathbf{\Lambda})$. Influence of $E O$ scions.

nante sur celle des racines (fig. 5) : l'écart entre une formule de greffage et le témoin autogreffé est systématiquement supérieur quand c'est le "greffon » qui est changé.

Les différences de résistance stomatique entre les essais pour une même formule de greffage (par exemple pour DOU/DOU dans les essais 1 et 4 : fig. 4) viennent sans doute de la variabilité des conditions cli- matiques durant la période d'élevage des plantes en serre. Mais on remarque que le greffage ne modifie en rien les différences écotypiques observées sur les plantes entières (fig. 2 et 3 ) : les autogreffes des variétés $\mathrm{EO}$ ont encore une résistance stomatique significativement plus faible que les autogreffes des variétés $\mathbf{M}$.

\section{Comportement au champ}

Aucune différence de résistance stomatique n'est observée entre les 6 variétés non greffées de l'expérience de 1981, ni entre les 12 combinaisons de greffage inter- et intra-écotypiques de 1982. Que le sol soit à la capacité de rétention ou plus sec juste avant une irrigation, les résistances stomatiques sont, dans les 2 cas, très faibles (de 0,2 à $3 \mathrm{~s} . \mathrm{cm}^{-1}$ ) dès $10 \mathrm{~h} \mathrm{du}$ matin. Quand la résistance stomatique commence à augmenter vers $19 \mathrm{~h}$, elle le fait de la même façon pour toutes les variétés (1981), ou combinaisons de greffage (1982).

\section{DISCUSSION}

Nous retrouvons la différence de comportement observée antérieurement entre des plantes en enceintes climatisées (POCHARD \& SERIEYS, 1974 ; POCHARD, 1979) et celles cultivées en plein champ (COLLECTIF, 1975 ; SERIEYS \& IGLESIAS, 1976 ; CORNILLON \& DAUPLE, 1981) : la résistance stomatique des variétés méditerranéennes est supérieure à celle des variét śs extrême-orientales en enceintes climatisées et sous serre, mais cette différence disparaît en culture de plein champ.

L'effet de la température de l'air (fig. 3a), ajouté à un léger effet possible de l'éclairement, explique ces résultats. A $20-22{ }^{\circ} \mathrm{C}$, c'est-à-dire aux températures courantes sous serre et dans des expériences en chambres climatisées (Pochard \& Serieys, 1974), les différences écotypiques sont nettes; mais elles s'estompent quand la température s'élève aux niveaux couramment observés l'été en plein champ $\left(30-35^{\circ} \mathrm{C}\right) \mathrm{car}$, à ces valeurs, la résistance stomatique devient presque nulle pour toutes les variétés. Au-delà de $35^{\circ} \mathrm{C}$, la régulation stomatique commence à intervenir : un peu plus énergiquement sur les variétés méditerranéennes si bien que la différence écotypique se rétablit. Ce type de réaction à la température est courant chez de nombreuses espèces (HOFSTRA \& HESKETT, 1969).

D'après l'expérience d'ombrage sous serre, les faibles éclairements que l'on a en chambres climatisées, pourraient exalter les différences écotypiques (fig. 1). Mais, dans ce type d'expérience, la diminution de l'éclairement est accompagnée d'une baisse simultanée de la température (cf. $\S$ II.B) qui augmente elle aussi les écarts entre les écotypes (fig. 3). L'effet de l'éclairement n'est donc pas certain et, en tout cas, il est probablement faible. Par ailleurs, de 100 (sous ombrage) à $700 \mathrm{~W} \cdot \mathrm{m}^{-2}$ (au champ), la résistance stomatique des faces inférieures reste toujours très faible. Il y a donc tout lieu de penser qu'elle est, à elle seule, représentative de la résistance "moyenne " des feuilles dans toute la gamme normale de lumière. 
Selon la règle générale (LANGE et al., 1971 ; FANJUL \& JONES, 1982, inter al.), la sécheresse de l'air augmente la résistance stomatique (fig. $3 \mathrm{~b}$ ) : le phénomène est net en dessous de $35^{\circ} \mathrm{C}$, dès que le déficit de saturation dépasse $5 \mathrm{mb}$. Mais au-delà de $35^{\circ} \mathrm{C}$, la réaction à la sécheresse de l'air dépend des variétés ; elle se comprend d'ailleurs difficilement sans l'intervention d'un phénomène qui dénature la réponse des stomates comme, par exemple, un stress hydrique (Ludlow, 1980). Or il est possible qu'à $40^{\circ} \mathrm{C}$ et un déficit de saturation de $27,5 \mathrm{mb}$, les quantités d'eau apportées n'aient pas correspondu à la demande de l'évapotranspiration potentielle, malgré les précautions prises.

Quoi qu'il en soit, même sous cette demande climatique, la régulation stomatique est encore faible pour toutes les variétés, puisqu'aucune d'entre elles n'atteint les $30 \mathrm{~s} . \mathrm{cm}^{-1}$ qui correspondent en général à la fermeture complète des stomates (de PARCEVAUX, 1964 ; TEARE et al., 1982). Par ailleurs, il est troublant que les variétés méditerranéennes aient, contre toute attente, des résistances stomatiques «plus faibles » en air sec qu'en air humide quand la température dépasse $35{ }^{\circ} \mathrm{C}$ (fig. 3), même si le phénomène a sans doute peu de poids sur le bilan transpiratoire. $\mathrm{Du}$ moins, ce résultat montre que l'adaptation de l'aubergine au climat sec méditerranéen ne vient certainement pas d'une plus grande sensibilité de la régulation stomatique qui diminuerait significativement le débit transpiratoire.

En bilan, la réaction à la température domine largement sur celle à la lumière et à l'humidité de l'air et explique très bien le changement de comportement des aubergines quand elles passent du climat " tempéré » d'une enceinte ou d'une serre à celui d'un été méditerranéen du sud de la France, où la température diurne est le plus souvent comprise entre 30 et $35^{\circ} \mathrm{C}$.

L'arrosage des plantes, toujours très abondant dans des expériences en chambres climatisées, place les racines dans des conditions proches d'une culture hydroponique. Or, TinKLIN \& WEATHERLEY (1966) et WEATHERLEY (1975) ont montré que, dans cette situation, la résistance la plus forte à la circulation de l'eau dans le système sol-plante-atmosphère, se situe au niveau des racines et qu'elle peut se répercuter au niveau des stomates. Aussi, peut-être les variations de résistance enregistrées au niveau des feuilles sont-elles en partie imputables aux variations de résistance des racines à la pénétration de l'eau, d'autant plus qu'en chambre climatisée, la température des substrats contenus dans les pots est probablement proche de celle de l'air. Or, sur tomates, DUPREY (1984) enregistre une augmentation de la résistance des racines à l'absorption de l'eau entre 33 et $37^{\circ} \mathrm{C}$ : on ne peut manquer de faire un rapprochement entre ces valeurs et le seuil de $35^{\circ} \mathrm{C}$ que nous avons enregistré sur les résistances stomatiques de l'aubergine, en chambre climatisée et en condition presque hydroponique.

Les réactions des greffes inter-écotypiques confirment cette influence des racines sur les résistances stomatiques en enceinte climatisée, puisque (fig. 4) les racines des variétés $\mathbf{M}$ l'augmentent et celles des $\mathrm{EO}$ la diminuent significativement. Cependant, l'influence des feuilles proprement dites, qui va strictement dans le même sens, est plus importante (fig. 5) : il faut donc tempérer les conclusions de TINKLIN \& WEA-
THERLEY (1966) et de Weatherley (1975). Par contre, on peut affirmer que la coordination de réactions entre les racines et les parties aériennes, déjà mise en évidence sur plusieurs paramètres de croissance (DAUNAY \& MALET, 1986 ; DAUNAY et al., 1986), intervient aussi sur un processus d'adaptation au climat comme la résistance stomatique.

Il est cependant probable que l'influence, déjà assez modeste, des racines s'estompe rapidement quand l'arrosage des plantes est moins abondant : c'était perut-être le cas dans l'expérience de greffage interécotypique de POCHARD \& SERIEYS (1974), dans laquelle aucune influence significative des racines n'était apparue.

On remarque que la variété RVA, appartenant à l'écotype EO mais cultivée depuis plusieurs décennies dans le bassin méditerranéen, a des réactions proches de celles de l'écotype méditerranéen. Ainsi les résistances stomatiques un peu plus fortes des variétés méditerranéennes en conditions presque hydroponiques sont peut-être une conséquence des processus d'adaptation aux conditions plus sèches.

En tout état de cause, le développement racinaire beaucoup plus important chez les variétés méditerranéennes fait certainement partie de ce processus. En effet, il leur permet d'absorber puis de transpirer plus d'eau (HALLAIRE, 1964 ; GARDNER, 1964 ; FEDDES \& RIJTEMA, 1972, inter al.). On retrouve ainsi la relation positive classique (ROBELIN, 1961 ; de PARCEVAUX, 1964) entre la quantité d'eau transpirée et la production de matière sèche. Car les variétés méditerranéennes ont bien les plus grandes surfaces foliaires et les plus fortes productions de fruits (DAUNAY \& MALET, 1986).

\section{CONCLUSION}

Quelle que soit la variété, un plant d'aubergine, bien alimenté en eau, a une régulation stomatique très peu active à la face inférieure des feuilles: presque insensible à la diminution de la lumière jusqu'à $50 \mathrm{~W} \cdot \mathrm{m}^{-2}$ et peu sensible à la sécheresse de l'air ; sa réaction la plus vive, mais toute relative, se fait quand les températures de l'air s'écartent, en diminuant ou en augmentant, de $35^{\circ} \mathrm{C}$, valeur caractéristique pour laquelle les stomates restent pratiquement grand ouverts, quel que soit le niveau des autres facteurs climatiques.

C'est la raison pour laquelle on commence à enregistrer une légère différence de comportement entre les 2 écotypes - une résistance stomatique un peu plus forte chez les variétés méditerranéennes - uniquement sous un faible éclairement, et dans un air ambiant à $20-25^{\circ} \mathrm{C}$ et à plus de $80 \mathrm{p} .100$ d'humidité relative. Dans ces conditions "protégées ", seulement possibles dans des serres ou des enceintes climatisées, cette différence vient sans doute d'une conductance de l'eau un peu moins bonne au niveau des racines, chez les variétés méditerranéennes; car les réactions des plantes greffées "inter-écotypiques » montrent que les racines ont une légère influence sur la résistance stomatique des feuilles.

Mais le léger handicap des variétés méditerranéennes disparaît complètement en culture de plein champ, sous climats chauds et secs et avec des régimes hydri- 
ques un peu moins réguliers. Les résistances stomatiques des 2 écotypes devenant aussi faibles les unes que les autres, les variétés méditerranéennes, grâce à leurs systèmes racinaires beaucoup plus développés et profonds, deviennent alors les plus aptes à répondre à une forte demande climatique d'évapotranspiration et comme cette capacité va de pair avec une meilleure photosynthèse, elles produisent des fruits plus lourds et plus nombreux.

Ainsi, la résistance des variétés méditerranéennes d'aubergine à la sécheresse du climat estival a pour originalité de ne pratiquement rien devoir à la régulation stomatique, car celle-ci intervient relativement peu sur le débit transpiratoire des plantes. Le fort développement de leur système racinaire explique en grande partie leur potentiel d'adaptation ; mais peutêtre s'y ajoute-t-il un processus de régulation des différences de potentiels hydriques entre les feuilles et les racines et/ou une résistance variable à la circulation de la sève dans les racines et les tiges ? Car ce sont deux autres mécanismes possibles pour réduire la transpiration des plantes. De telles hypothèses méritent des expériences nouvelles.

Recule 21 juin 1985 Accepté le 11 mars 1986.

\section{RÉFÉRENCES BIBLIOGRAPHIQUES}

Beyries A.. 1979. Le greffage, moven de lutte contre les parasites telluriques des Solanees cultivees pour leurs fruits. Thèse Doct. Univ., mention Sci. Nat., Univ. Sci. Tech. Languedoc, Montpellier, $166 \mathrm{p}$.

Collectif, 1975. Modilités d'apport d'eau aux cultures. C. R. 1975 cd. 1976, 66 p., Groupe de travail plante el climat, Cons. Sci. du Cent. de Recl, d'Avignon, INRA, 84140 Montfavet, France.

Cornillon P., Dauplé P., 1981. Influence of irrigation rhythm and water supplv on growth, water status and vield of eggplant (Solanum melongena L.). Plant and Soil. 59, 365-379.

Daunay M. C.. 1982. Développement de l'aubergine et aduptation au climat: étude préliminaire. Rapp. de DEA, Univ. d'Aix Marseille III, éd. Sin. d'Amélior. Plantes Maraîchères, INRA, 84140 Montfavet, France, 22 p.

Daunay M. C., Malet Ph., 1986. Influences réciproques des racines el des parties aćriennes sur le rendement de l'aubergine (Solamam melongena L.) : Résultats de greffages inter-variétaux. Agronomic'. 6 (3), 293-297.

Daunay M. C.. Schoch P. G., Malet P., 1986. Fréquences s10matiques de l'aubergine (Solanum melongena $\mathrm{L}$.) et relations avec le développement racinaire. Agronomie, 6 (6).

Duprey Y., 1984. Contribution a l'étucte des effets des haute's $1 \mathrm{~cm}$ pératures racinaires sur la croissance de la tomate (Lycopersicon esculentum Mill.). Aspects particuliers concernant la nutrition $a_{\mathbf{n}} \mathrm{O}-$ tée. Rapp. de DEA, Univ. des Sci. et Tech. du Languedoc, Montpellier, Ed. Stn. d'Agron., INRA, 84140 Montfavet, France, $70 \mathrm{p}$

Fanjul L., Jones H. G.. 1982. Rapid stomatal responses to humidity. Planta, 154, 135-138.

Feddes R. A., Rijtema P. E., 1972. Water withdrawal by plant roots. J. of Hydrol., 17, 33-59.

Gardner W. R., 1964. Relation of root distribution to water uplake and availability. Agron. J., 56, 35-41.

Hallaire M.. 1964. Le potentiel efficace de l'eau dans le sol en régime de dessèchement, 27-62. In : L'eau et la production végélale », éd. INRA, $455 \mathrm{p}$
Hofstra G., Hesketh J. D., 1969. The effect of temperature on sto matal aperture in different species. Can. J. Bot., 47. 1307-1310.

Lange O. L., Lösch R., Schultze E. D., Kappen L., 1971. Responses of stomata to clianges in humidity. Planta, 100, 76-86.

Ludlow M. M., 1980. Adaptative significance of stomatal responses to water stress, 123-138. In : N. C. Turner \& P. J. Kramer: "Adtaptation of plants to water and high temperature stress ", Whiley interscience publ., $475 \mathrm{p}$.

Parcevaux S. de. 1964. Transpiration vegétale et production de matière sèche, essai d'interprétation en fonction des facteurs du milieu, 63-150. In : "L'eau et la production végétale ", éd. INRA, $455 \mathrm{p}$.

Pochard E., 1979. Adaptation climatique et proprietes hydriques, réponse de l'aubergine au climal des serres. Riv. di Agron., anno XIII, 3, 381-391.

Pochard E., Serieys H., 1974. Mise en évidence de différences varie tales de transpiration che? l'aubergine et d'autres solanacées (Solanum melongena L., Capsicum annuum L., Lycopersicon esculentum Mill.), conséquences pour l'adaptation à différents climats. Ann. Amélior. Plantes, 24 (3), 243-268.

Robelin M., 1961. Eléments sur la rentabilite de l'irrigation. Ann. Agron., 12 (1), 65-73:

Serieys H., Iglesias A. P., 1976. Evolution journalière de la résistance stomatique, 29-30. In : Modalités d'apport d'eau alt.x cultures », C. R. 1976, groupe de trav, plante et climat, Cons. Sci. du Cent. de Rech. d'Avignon. INRA, 84140 Montfavet, France.

Teare I. D., Nasser Sionit, Kramer P. J., 1982. Changes in water status during water stress at different stages of development in wheat. Physiol. Plant., 55, 296-300.

Tinklin R., Weatherley P. E.. 1966. On the relationship between transpiration rate and leaf water potential. New Phvtol., 65. 509517.

Weatherley P. E., 1975. Water relations of the root system, 397-413. In : J. G. Torrey \& D. T. Clarkson : «The development and function of roots". Academic Press, $611 \mathrm{p}$. 